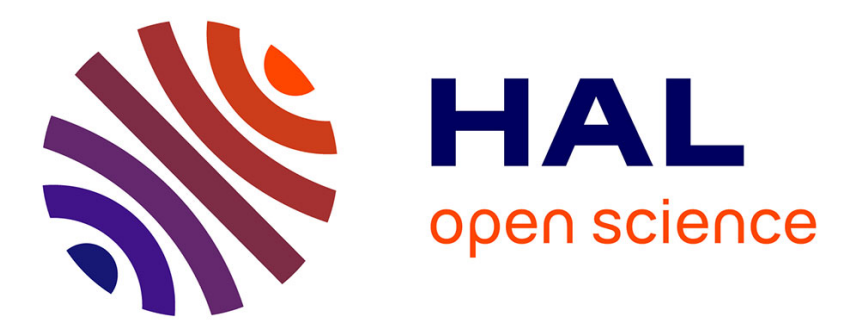

\title{
Nonlinear optical diffraction by standing acoustic waves in a GaAs film
}

\author{
N.A. Shevchenko, N.N. Dadoenkova, I.L. Lyubchanskii, F.F.L. Bentivegna, \\ Y.P. Lee, Th. Rasing
}

\section{- To cite this version:}

N.A. Shevchenko, N.N. Dadoenkova, I.L. Lyubchanskii, F.F.L. Bentivegna, Y.P. Lee, et al.. Nonlinear optical diffraction by standing acoustic waves in a GaAs film. Photonics and Nanostructures Fundamentals and Applications, 2012, 10, pp.400. 10.1016/j.photonics.2012.05.003 . hal-00938971

\section{HAL Id: hal-00938971 https://hal.science/hal-00938971}

Submitted on 29 Jan 2014

HAL is a multi-disciplinary open access archive for the deposit and dissemination of scientific research documents, whether they are published or not. The documents may come from teaching and research institutions in France or abroad, or from public or private research centers.
L'archive ouverte pluridisciplinaire HAL, est destinée au dépôt et à la diffusion de documents scientifiques de niveau recherche, publiés ou non, émanant des établissements d'enseignement et de recherche français ou étrangers, des laboratoires publics ou privés. 


\title{
Nonlinear Optical Diffraction by \\ Standing Acoustic Waves in a GaAs film
}

\author{
N.A. Shevchenko ${ }^{1}$, N.N. Dadoenkova ${ }^{1}$, I.L. Lyubchanskii ${ }^{1,2}$, \\ F.F.L. Bentivegna ${ }^{3}$, Y.P. Lee ${ }^{4}$, and Th. Rasing ${ }^{5}$ \\ ${ }^{1}$ Donetsk Physical \& Technical Institute of the National Academy of Sciences of Ukraine, 72, R. Luxemburg str., \\ 83114, Donetsk, Ukraine \\ ${ }^{2}$ Department of Physics and Technology, Donetsk National University, 13, Theatralny ave., 83001, Donetsk, Ukraine \\ ${ }^{3}$ Lab-STICC (UMR CNRS 6285), ENIB - Université Européenne de Bretagne, CS 73862, 29238 Brest Cedex 3, \\ France \\ ${ }^{4}$ Quantum Photonic Science Research Center and Department of Physics, Hanyang University, \\ 17 Haendang-Dong, Sundong-Ku, Seoul 133 791, Korea \\ ${ }^{5}$ Institute for Molecules and Materials, Radboud University Nijmegen, Heyendaalseweg 135, \\ 6525 AJ Nijmegen, The Netherlands
}

\begin{abstract}
Second-order nonlinear optical diffraction by standing acoustic waves in a crystalline plate is theoretically investigated. A detailed analysis of the polarization state of the second-harmonic light diffracted by both longitudinal and transversal acoustic waves is carried out. It is shown that longitudinal standing acoustic waves only allow p-polarized nonlinear optical diffraction, irrespective of the incoming state of polarization, whereas transversal standing acoustic waves allow all possible combinations of incoming and diffracted polarization states. Numerical estimates of the relative intensities of nonlinearly diffracted radiation peaks are made for a GaAs plate.
\end{abstract}

Keywords: Quadratic optical nonlinearity; Modulated structures; Nonlinear optical diffraction; Standing acoustic waves. 


\section{Introduction}

The phenomenon of optical diffraction from periodically modulated structures (e.g., diffraction gratings) is well known in classical optics [1] and is widely used for a broad range of optical devices [2]. If in addition such a medium exhibits nonlinear optical properties, observation of nonlinear optical diffraction (NOD), i.e., diffraction at higher harmonics of the incident frequency, is possible provided certain conditions are fulfilled. In media with a quadratic optical nonlinearity, NOD is thus expected to be observed at a frequency double that of the incident electromagnetic wave (EMW). The first theoretical and experimental investigation of quadratic NOD was made in a periodically poled ferroelectric $\mathrm{NH}_{4} \mathrm{Cl}$ crystal, where the modulation period was comparable with the wavelengths of the second harmonic EMWs generated from the periodic structure [3]. Further theoretical and experimental studies of NOD were carried out in such modulated structures as ordered magnetic domain arrays [4-7] and photonic crystals [813]. One of the possible ways to modulate the nonlinear polarization in a crystalline medium is to excite a standing acoustic wave characterized by a periodically distributed displacement field in the sample with spatially localized nodes and antinodes. In linear optics, a similar phenomenon is well known as light diffraction by ultrasound waves [1, 14]. In the case of NOD, the nonlinear polarization of the medium will be modulated by the deformations of the crystal through a nonlinear photoelastic interaction. Such a situation was discussed in the frame of acoustically-induced optical second harmonic generation (SHG) due to the interaction between optical waves and a travelling acoustic wave in a crystal [15]. Some theoretical aspects of nonlinear photoelastic interaction were discussed by several groups [16 - 18]. The effect of acoustically-induced optical SHG was also observed in GaAs films in the infrared region where this material is transparent [19]. Later, strain-induced three-photon effects such as optical SHG in strained crystals and hyper-Rayleigh light scattering by dislocation deformation were theoretically investigated [20], as well as SHG due to misfit strain and misfit dislocations at filmsubstrate interfaces [21]. Similarly, experimental observations of strain-induced SHG were reported in silicon wafers [22] and the influence of strain on SHG in $\mathrm{Co}_{0.25} \mathrm{Pd}_{0.75}$ films on a PZT substrate [23], in bismuth-substituted iron garnet films [24], as well as in bulk silicon [25, 26] were discussed.

In this paper, we theoretically investigate NOD induced by standing longitudinal and transversal acoustical waves in a crystalline plate. This situation differs from the aforementioned works [15-19] insofar as it does not require the same phase matching conditions to be fulfilled. The paper is organized as follows. In Section 2 we describe strain-induced nonlinear polarization on the basis of the photoelastic interaction, and Section 3 introduces the mechanical strain originating in standing acoustic waves in a crystalline film. Section 4 is devoted to the analysis 
of second-order NOD by both longitudinal and transversal standing acoustic waves in such a film and presents and discusses the results of numerical simulations carried out in the case of a GaAs film.

\section{Strain-induced nonlinear polarization}

In the dipole approximation the quadratic nonlinear optical polarization vector $\mathbf{P}^{\mathrm{NL}}(2 \omega)$ can be written in a Cartesian referential as [27]:

$$
P_{i}^{N L}(2 \omega)=\varepsilon_{0} \chi_{i j k}^{(2)}(-2 \omega: \omega, \omega) E_{j}(\omega) E_{k}(\omega)
$$

where $\mathbf{E}(\omega)$ is the electric field of the incident EMW at frequency $\omega, \chi_{i j k}^{(2)}$ is the quadratic nonlinear optical susceptibility (NOS) tensor of the medium, and $\varepsilon_{0}$ is the vacuum permittivity. When a spatial modulation of the optical properties of the medium takes place - e.g., due to the presence of a standing acoustic wave - the NOS tensor can be expressed as:

$$
\chi_{i j k}^{(2)}(\mathbf{r})=\chi_{i j k}^{(2,0)}(\mathbf{r})+p_{i j k l m} u_{l m}(\mathbf{r})=\chi_{i j k}^{(2,0)}(\mathbf{r})+\left(\frac{\partial \chi_{i j k}^{(2)}}{\partial u_{l m}}\right)_{u_{l m}=0} u_{l m}(\mathbf{r}),
$$

where $\chi_{i j k}^{(2,0)}$ is the nonlinear optical susceptibility tensor in the absence of modulation, $p_{i j k l m}$ is the nonlinear photoelastic tensor and $u_{l m}$ is the strain tensor. In the linear approximation, the latter is symmetric, i.e. $u_{l m}=u_{m l}$. The tensor $p_{i j k l m}$ was first introduced in Ref. [15] for the description of acoustically-induced optical SHG. From Eq. (2) it follows that the symmetry properties of the nonlinear photoelastic tensor $p_{i j k l m}$ derive from those of both the NOS tensor $\chi_{i j k}^{(2)}$ and the strain tensor $u_{l m}$. Thus the symmetry of the strain tensor imply that $p_{i j k l m}=p_{i k j l m}=p_{i j k m l}=p_{i k j m l}$, which strongly reduces the number of independent components of the NOS tensor $\chi_{i j k}^{(2)}$. In addition, that tensor has non-zero components in non-centrosymmetric crystals only, i.e., in media without inversion center [27].

The second-harmonic electric field $\mathbf{E}_{2 \omega}(\mathbf{r}, t)$ emitted from a non-magnetic medium is a solution of the quadratic nonlinear wave equation which, in SI units, writes [27]:

$$
\nabla \times \nabla \times \mathbf{E}_{2 \omega}(\mathbf{r}, t)+\frac{n_{2 \omega}^{2}}{c^{2}} \frac{\partial^{2} \mathbf{E}_{2 \omega}(\mathbf{r}, t)}{\partial t^{2}}=-\mu_{0} \frac{\partial^{2} \mathbf{P}_{2 \omega}^{\mathrm{NL}}(\mathbf{r}, t)}{\partial t^{2}},
$$

where $n_{2 \omega}$ is the refractive index of the medium at the second harmonic frequency, $c$ is the velocity of light in vacuum, and $\mu_{0}$ is the vacuum permeability. Taking the Fourier transform of Eq. (3) with respect to time and using the slowly varying envelope approximation, where $\mathbf{k}^{2 \omega} \cdot \nabla \mathbf{E}(2 \omega, \mathbf{r}) \gg \Delta \mathbf{E}(2 \omega, \mathbf{r})$, leads to the following reduced form [27, 28]: 


$$
\mathbf{k}^{2 \omega} \cdot \nabla \mathbf{E}(2 \omega, \mathbf{r})=-2 i \frac{\omega^{2}}{c^{2}} \mathbf{P}^{\mathrm{NL}}(2 \omega, \mathbf{r}) \exp (i \mathbf{q} \cdot \mathbf{r})
$$

Here $\mathbf{E}(2 \omega, \mathbf{r})$ and $\mathbf{P}^{\mathrm{NL}}(2 \omega, \mathbf{r})$ are the temporal Fourier transforms of the electric field at the second harmonic frequency and the corresponding nonlinear polarization, and $\mathbf{q}=2 \mathbf{k}^{\omega}-\mathbf{k}^{2 \omega}$ is the phase-mismatch vector, while $\mathbf{k}^{\omega}$ and $\mathbf{k}^{2 \omega}$ are the wave vectors of the fundamental and second harmonic EMWs, respectively. The solution of wave equation (4) in the plane-wave approximation can then be expressed as [27, 28]:

$$
\mathbf{E}(2 \omega, \mathbf{q})=\frac{i \omega}{c n_{2 \omega}} \frac{1}{V} \int_{V} \mathbf{P}^{\mathrm{NL}}(2 \omega, \mathbf{r}) \exp (i \mathbf{q} \cdot \mathbf{r}) d \mathbf{r},
$$

The integral is taken over the interaction volume $V$, i.e., in the irradiated region where the electric field of the incident EMW is non-zero.

\section{Strain induced by standing acoustic waves}

We now consider a non-centrosymmetric thin crystalline film whose surfaces are perpendicular to the $y$-axis of a (xyz) Cartesian referential (Fig. 1). In what follows, calculations will be made for a GaAs film, whose zinc blende crystal structure (with a $\overline{4} 3 m$ or $T_{d}$ point group symmetry) is indeed non-centrosymmetric. We suppose the system to be continuous with respect to its elastic properties. The form of standing acoustic waves in a bulk elastic medium can be deduced from the following system of equations of motion [29]:

$$
\rho \frac{\partial^{2} u_{i}(\mathbf{r}, t)}{\partial t^{2}}=c_{i j k l} \frac{\partial^{2} u_{l}(\mathbf{r}, t)}{\partial r_{j} \partial r_{k}},
$$

where $\rho$ and $c_{i j k l}$ are the density of the crystal and the elastic stiffness tensor, respectively, and the summation convention over repeated indices is assumed. For cubic crystals without inversion center, only three components of the $c_{i j k l}$ tensor are independent [29]:

$$
c_{11} \equiv c_{x x x x}=c_{y y y y}=c_{z z z z}, c_{12} \equiv c_{x x y y}=c_{y y z z}=c_{z z x x}, c_{44} \equiv c_{x y x y}=c_{y z y z}=c_{z x z x} .
$$

The same symmetry rules hold for the zinc blende structure. Consequently, Eq. (6) reduces to the following system:

$$
\begin{aligned}
& \rho \frac{\partial^{2} u_{x}}{\partial t^{2}}=c_{11} \frac{\partial^{2} u_{x}}{\partial x^{2}}+c_{44}\left(\frac{\partial^{2} u_{x}}{\partial y^{2}}+\frac{\partial^{2} u_{x}}{\partial z^{2}}\right)+\left(c_{12}+c_{44}\right)\left(\frac{\partial^{2} u_{y}}{\partial x \partial y}+\frac{\partial^{2} u_{z}}{\partial x \partial z}\right), \\
& \rho \frac{\partial^{2} u_{y}}{\partial t^{2}}=c_{11} \frac{\partial^{2} u_{y}}{\partial y^{2}}+c_{44}\left(\frac{\partial^{2} u_{y}}{\partial x^{2}}+\frac{\partial^{2} u_{y}}{\partial z^{2}}\right)+\left(c_{12}+c_{44}\right)\left(\frac{\partial^{2} u_{z}}{\partial y \partial z}+\frac{\partial^{2} u_{x}}{\partial x \partial y}\right),
\end{aligned}
$$




$$
\rho \frac{\partial^{2} u_{z}}{\partial t^{2}}=c_{11} \frac{\partial^{2} u_{z}}{\partial z^{2}}+c_{44}\left(\frac{\partial^{2} u_{z}}{\partial y^{2}}+\frac{\partial^{2} u_{z}}{\partial x^{2}}\right)+\left(c_{12}+c_{44}\right)\left(\frac{\partial^{2} u_{x}}{\partial z \partial x}+\frac{\partial^{2} u_{y}}{\partial y \partial z}\right) .
$$

The acoustic waves generated within the crystal are assumed to propagate along the $z$-axis of the referential (Fig. 1), which is also the four-fold [001] axis of the crystal. Thus the mechanical displacement vector $\mathbf{u}$ is function of the sole $z$ spatial coordinate and of time $t$. Hence, Eqs. (8) simplify to the following set of wave equations:

$$
\rho \frac{\partial^{2} u_{x}}{\partial t^{2}}=c_{44} \frac{\partial^{2} u_{x}}{\partial z^{2}}, \quad \rho \frac{\partial^{2} u_{y}}{\partial t^{2}}=c_{44} \frac{\partial^{2} u_{y}}{\partial z^{2}}, \quad \rho \frac{\partial^{2} u_{z}}{\partial t^{2}}=c_{11} \frac{\partial^{2} u_{z}}{\partial z^{2}} .
$$

It can be seen from Eqs. (9) that in such a crystal an acoustic wave excited along the $z$-axis actually consists of two components propagating independently with different phase velocities. The displacement $u_{z}(z, t)$ associated to the first component is parallel to the direction of excitation and propagates with the velocity $v_{L}=\left[c_{11} / \rho\right]^{1 / 2}-$ this component is a so-called longitudinal wave ( $L$-wave). The displacement $\left[u_{x}(z, t), u_{y}(z, t)\right]$ associated to the second component is perpendicular to the direction of excitation and propagates with a phase velocity $v_{T}=\left[c_{44} / \rho\right]^{1 / 2}-$ that component is a transverse wave ( $T$-wave). Thus the overall general expression for the displacement vector, $\mathbf{u}(z, t)=\mathbf{u}_{L}(z, t)+\mathbf{u}_{T}(z, t)$, writes as the sum of a longitudinal and a transverse part, each of which satisfies the wave equation with the appropriate velocity.

One possible solution of Eqs. (9) in the bulk of the crystal is a standing acoustic wave that results from the interference between two traveling waves propagating in opposite directions - as is bound to happen in a crystal plate whose extension along the $z$-axis is finite. The displacement vector for the bulk standing acoustic wave can then be written as:

$$
\mathbf{u}(z, t)=\mathbf{A}_{L} \cos \left(\frac{\Omega_{L} z}{v_{L}}\right) \exp \left(i \Omega_{L} t\right)+\mathbf{A}_{T} \cos \left(\frac{\Omega_{T} z}{v_{T}}\right) \exp \left(i \Omega_{T} t\right) .
$$

Here $\mathbf{A}_{L}=\left[0,0, A_{z}\right]$ and $\mathbf{A}_{T}=\left[A_{x}, A_{y}, 0\right]$ are the amplitudes of the longitudinal and transverse acoustic standing waves, respectively, and $\Omega_{L, T}=\frac{\pi v_{L, T}}{L_{z}}$, are their frequencies, where $L_{z}$ is the length of the crystal along the $z$-axis. For standing waves, there is no propagation of the mechanical energy along that axis, i.e., the amplitudes of the displacement vector vary with position $z$ and time $t$ but their extrema are stationary. Values of $z$ obeying the conditions $\left(\Omega_{L, T} z\right) / v_{L, T}=(m+1 / 2) \pi, m \in \mathbb{N}$, denote the nodes of the vibration, i.e., points in the crystal where the vibration is zero for all values of $t$, whereas the amplitudes of the vibration are 
maximal at any time $t$ at its antinodes, for which $\left(\Omega_{L, T} z\right) / v_{L, T}=m \pi, m \in \mathbb{N}$. As usual with standing waves, the distance between successive nodes or antinodes is equal to half the wavelength of the exciting acoustic wave (Fig. 1).

In practice, such transversal and longitudinal acoustical waves can be excited in thin films and plates with piezoelectric transducers, as thoroughly described for instance in Ref. [14]. It is also possible to use optical methods to excite both travelling and standing acoustical waves in bulk materials as well as in thin films and multilayers [30].

\section{Nonlinear diffraction by standing acoustic waves}

The plane of incidence of light is taken to be the $(y z)$-plane. The wave vectors of the secondharmonic EMWs diffracted from the crystal must satisfy the nonlinear Bragg condition $\mathbf{k}^{2 \omega}=2 \mathbf{k}^{\omega}+N \mathbf{Q}_{L, T}, N \in \mathbb{Z}$ for three-wave interaction, where $\mathbf{Q}_{L}$ and $\mathbf{Q}_{T}$ are the wave vectors of the longitudinal and transverse standing acoustic waves, respectively $[3,4-6,8,9]$. Alternatively, that vectorial equality can be decomposed in two scalar conditions for in-plane and out-of-plane wave vector components, respectively:

$$
\begin{gathered}
k^{2 \omega} \sin \left[\varphi_{2 \omega}^{(N)}\right]=2 k^{\omega} \sin \left(\varphi_{\omega}\right)+N Q_{L, T}, \\
k^{2 \omega} \cos \left[\varphi_{2 \omega}^{(N)}\right]=2 k^{\omega} \cos \left(\varphi_{\omega}\right) .
\end{gathered}
$$

Here angles $\varphi_{\omega}$ and $\varphi_{2 \omega}^{(N)}$ refer to the directions of the incident and $N$-th order diffracted EMWs, respectively (Fig. 1), and $Q_{L}=\left[\left(\Omega_{L}^{2} \rho\right) / c_{11}\right]^{1 / 2}$ and $Q_{T}=\left[\left(\Omega_{T}^{2} \rho\right) / c_{44}\right]^{1 / 2}$ are the wavenumbers of the $L$ - and $T$-standing acoustic waves in the crystal. From Eq. (11a) one can derive the maximum number of diffraction orders that can be observed in each case:

$$
N_{\max }^{L, T}=\frac{\Lambda_{L, T}}{\lambda_{2 \omega}}\left[n_{2 \omega}-n_{\omega} \sin \left(\varphi_{\omega}\right)\right] .
$$

In Eq. (12) $\Lambda_{L, T}$ are the wavelengths of $L$ - and $T$-standing acoustic waves, respectively, $\lambda_{2 \omega}$ is the wavelength in vacuum of the diffracted EMW at frequency $2 \omega$, and $n_{\omega}$ and $n_{2 \omega}$ are the refractive indexes of the crystal at the fundamental and second harmonic frequencies. Figure 2 shows the dependence of $N_{\max }^{L, T}$ as functions of the incidence angle $\varphi_{\omega}$ for various values $(250 \mathrm{MHz}, 500 \mathrm{MHz}$, and $1 \mathrm{GHz})$ of the acoustic wave frequencies $F_{L, T}=\frac{v_{L, T}}{2 L_{z}}$. Calculations are made for GaAs at the $\mathrm{CO}_{2}$ laser wavelength $\lambda_{\omega}=10.6 \mu \mathrm{m}$.

The efficiency of NOD induced by standing acoustic waves strongly depends on the polarization state of light. For a given phase-mismatch vector in the crystal, the electric fields 
$\mathbf{E}_{s, p}(2 \omega, \mathbf{q})$ of second-harmonic $s$ - and $p$-polarized EMWs are phenomenologically related to those at the fundamental frequency through the second-order NOS tensor as follows:

$$
\left[\begin{array}{l}
\mathbf{E}_{s}(2 \omega, \mathbf{q}) \\
\mathbf{E}_{p}(2 \omega, \mathbf{q})
\end{array}\right]=-\frac{i \omega}{c n_{2 \omega}}\left[\begin{array}{ll}
\chi_{s ; s s}^{(2)}(\mathbf{q}) & \chi_{s ; p p}^{(2)}(\mathbf{q}) \\
\chi_{p ; s s}^{(2)}(\mathbf{q}) & \chi_{p ; p p}^{(2)}(\mathbf{q})
\end{array}\right] \cdot\left[\begin{array}{l}
\mathbf{E}_{s}^{2}(\omega) \\
\mathbf{E}_{p}^{2}(\omega)
\end{array}\right] .
$$

The components of that tensor depend on those of both the nonlinear photoelastic tensor and the deformation tensor of the crystal in the spatial frequency domain, hence they differ depending on the nature of the standing acoustic wave responsible for nonlinear diffraction. Taking into account the symmetries of GaAs ( $T_{d}$ point group), they are, for $L$-acoustic waves:

$$
\begin{gathered}
\chi_{s ; s s}^{(2, N)}(\mathbf{q})=0, \\
\chi_{p ; s s}^{(2, N)}(\mathbf{q})=\frac{p_{z x x z z}}{\cos \left(\varphi_{2 \omega}^{(N)}\right)} u_{z z}(\mathbf{q}), \\
\chi_{s ; p p}^{(2, N)}(\mathbf{q})=0, \\
\chi_{p ; p p}^{(2, N)}(\mathbf{q})=\left[p_{y y z z z} \sin \left(2 \varphi_{\omega}\right) \sin \left(\varphi_{2 \omega}^{(N)}\right)+\left(p_{z y y z z} \sin ^{2}\left(\varphi_{\omega}\right)+p_{z z z z z} \cos ^{2}\left(\varphi_{\omega}\right)\right) \cos \left(\varphi_{2 \omega}^{(N)}\right)\right] u_{z z}(\mathbf{q}) ;
\end{gathered}
$$

and for $T$-acoustic waves:

$$
\begin{gathered}
\chi_{s ; s s}^{(2, N)}(\mathbf{q})=p_{x x x x z} u_{x z}(\mathbf{q}), \\
\chi_{p ; s s}^{(2, N)}(\mathbf{q})=\frac{p_{y x x y z}}{\sin \left(\varphi_{2 \omega}^{(N)}\right)} u_{y z}(\mathbf{q}), \\
\chi_{s ; p p}^{(2, N)}(\mathbf{q})=\left[p_{x y y x z} \sin ^{2}\left(\varphi_{\omega}\right)+p_{x z z x z} \cos ^{2}\left(\varphi_{\omega}\right)\right] u_{x z}(\mathbf{q}), \\
\chi_{p ; p p}^{(2, N)}(\mathbf{q})=\left[\left(p_{y y y y z} \sin ^{2}\left(\varphi_{\omega}\right)+p_{y z z y z} \cos ^{2}\left(\varphi_{\omega}\right)\right) \sin \left(\varphi_{2 \omega}^{(N)}\right)+p_{z y z y z} \sin \left(2 \varphi_{2 \omega}^{(N)}\right) \cos \left(\varphi_{2 \omega}^{(N)}\right)\right] u_{y z}(\mathbf{q}) .
\end{gathered}
$$

The components of the strain tensor in the frequency domain that appear in Eqs. (14) - (15) are obtained by Fourier transform from their counterparts in real space, with:

$$
u_{l m}(\mathbf{q})=\frac{1}{V} \int_{V} u_{l m}(\mathbf{r}) \exp (i \mathbf{q} \cdot \mathbf{r}) d \mathbf{r}=I\left(q_{\|}\right) I_{l m}\left(q_{\perp}\right),
$$

where integration in Eq. (16a) is taken over the irradiated volume, $q_{\|}$and $q_{\perp}$ are the longitudinal and transversal components of wave vector $\mathbf{q}$, and $I\left(q_{\|}\right)$and $I_{l m}\left(q_{\perp}\right)$ are integrals defined as:

$$
I\left(q_{\|}\right)=\frac{1}{H} \int_{-H / 2}^{H / 2} \exp \left(i q_{\|} z\right) d z=\operatorname{sinc}\left(\frac{q_{\|} H}{2}\right),
$$




$$
\begin{aligned}
I_{l m}\left(q_{\perp}\right) & =\frac{1}{\pi R^{2}} \int_{0}^{R} u_{l m}(\rho) \rho d \rho \int_{0}^{2 \pi} \exp \left(i q_{\perp} \rho \cos \varphi\right) d \varphi \\
& =2 i\left(A_{l} Q_{L, T ; m}+A_{m} Q_{L, T ; l}\right)\left[\frac{J_{1}\left[\left(q_{\perp}+N \frac{\Omega_{L, T}}{v_{L, T}}\right) R\right]}{\left(q_{\perp}+N \frac{\Omega_{L, T}}{v_{L, T}}\right) R}-\frac{J_{1}\left[\left(q_{\perp}-N \frac{\Omega_{L, T}}{v_{L, T}}\right) R\right]}{\left(q_{\perp}-N \frac{\Omega_{L, T}}{v_{L, T}}\right) R}\right] .
\end{aligned}
$$

In Eq. (16b), integral $I\left(q_{\|}\right)$is evaluated over the film thickness $H, \operatorname{sinc}(x) \equiv \sin (x) / x$, and $q_{\|}=2 \pi / \lambda_{2 \omega}\left[n_{\omega} \cos \left(\varphi_{\omega}\right)-n_{2 \omega} \cos \left(\varphi_{2 \omega}\right)\right]$. In Eq. (16c), integral $I_{l m}\left(q_{\perp}\right)$ is calculated over the cross-section of the irradiating spot of radius $R$ at the film surface, whose points are referred to by their polar coordinates $\rho$ and $\varphi$ in the $(x z)$-plane, $J_{1}(x)$ is the first-order Bessel function of the first kind, and $q_{\perp}=2 \pi / \lambda_{2 \omega}\left[n_{\omega} \sin \left(\varphi_{\omega}\right)-n_{2 \omega} \sin \left(\varphi_{2 \omega}\right)\right]$. For the sake of clarity, the order of diffraction $N$, upon which the angle of diffraction at $2 \omega$ depends, was not explicitly mentioned in Eqs. (16) and in the expressions of $q_{\|}$and $q_{\perp}$.

From Eqs. (14) - (15), it appears that $L$-acoustic waves can only give rise to $p$-polarized nonlinear diffraction, whatever the state of polarization of the incoming beam, whereas $T$ acoustic waves allow all four types $(s \rightarrow s, s \rightarrow p, p \rightarrow s$, and $p \rightarrow p$ ) of nonlinear diffraction processes. In order to estimate the relative intensities of the second-harmonic diffracted beams in each configuration, one can introduce nonlinear diffraction efficiencies $D_{\alpha, \beta \beta}^{N O D}$ defined as

$$
D_{\alpha, \beta \beta}^{N O D}=\frac{I(2 \omega)}{I^{2}(\omega)} \propto\left|\chi_{\alpha, \beta \beta}^{(2)}(\mathbf{q})\right|^{2}, \quad\{\alpha, \beta\} \in(\{s, s\},\{s, p\},\{p, s\},\{p, p\}),
$$

where $I(2 \omega)$ and $I(\omega)$ are the intensities of the diffracted and incident beams. Again, the order of diffraction $N$ has been omitted in Eq. (17).

Figure 3 depicts the angular dependencies of the diffraction efficiencies $D_{\alpha, \beta \beta}^{N O D}$ for $L$ - and $T$ - standing acoustic waves for the first order of nonlinear diffraction $(N=1)$, calculated using Eqs. (14) - (15) and the numerical values of the nonlinear photoelastic tensor components of GaAs taken from Ref. [18], for the following combinations of polarization state of incident and diffracted second-harmonic EMWs: $\quad s(\omega) \rightarrow p(2 \omega), \quad p(\omega) \rightarrow p(2 \omega), \quad s(\omega) \rightarrow s(2 \omega)$ and $p(\omega) \rightarrow s(2 \omega)$. In accordance with Eqs. (14), (15) and (17), only the first two combinations yield any NOD for $L$-acoustic waves, whereas all four are possible with $T$-acoustic waves. As can be seen on Fig. 3, the maximal efficiency of the NOD process should be observed for a $p$ polarized incident EMW in the case of diffraction by $L$-acoustic waves. Similarly, the diffraction of $s$-polarized EMWs by $T$-acoustic waves is less efficient than that of $p$-polarized EMWs. Conversely, the results shown on the example of GaAs at $10.6 \mu \mathrm{m}$ suggest a potential use of 
NOD for the determination of the components of the nonlinear photoelastic tensor of that crystal - and of any crystal with similar symmetries - as a function of wavelength. With that application in mind, the approach developed in this paper can thus serve as a guide for the choice of the best combination of polarization states and angles of both the incident and the diffracted fields.

\section{Conclusions}

In this paper, we proposed a full theoretical analysis of second-order nonlinear optical diffraction due to the interaction of electromagnetic waves with both longitudinal and transverse standing acoustic waves in a GaAs crystal. We showed that this effect strongly depends on the polarization state of light. In particular, longitudinal standing acoustic waves only allow ppolarized nonlinear optical diffraction, whatever the incoming state of polarization, whereas transversal standing acoustic waves allow all possible polarization combinations of incoming and diffracted polarization states. A potential application of nonlinear optical diffraction from standing acoustic waves in crystals is the determination of the non-zero components of their nonlinear photoelastic tensor.

\section{Acknowledgments}

This work was supported by the FP7-People-2009-IRSES Project "NOWAPHEN" No. 247556 (N. A. Shevchenko, N. N. Dadoenkova, and I. L. Lyubchanskii), the Ministry of Education, Science and Technology (MEST) and the National Research Foundation (NRF) of South Korea through q-Psi (Y. P. Lee), and the Netherlands Organization for Scientific Research (NWO, Th. Rasing). 


\section{References}

[1] M. Born, E. Wolf, Principles of Optics, seventh ed., Cambridge University Press, Cambridge, 1999.

[2] C. Palmer, E.G. Loewen, Diffraction Grating Handbook, sixth ed., Newport Corporation, New York, 2005.

[3] I. Freund, Nonlinear diffraction, Phys. Rev. Lett. 21 (1968) 1404-1406.

[4] N. N. Dadoenkova, I. L. Lyubchanskii, M. I. Lyubchanskii, Th. Rasing, Nonlinear magnetooptical diffraction from periodic domain structures in magnetic films, Appl. Phys. Lett. 74 (1999) 1880-1882.

[5] I. L. Lyubchanskii, N. N. Dadoenkova, M. I. Lyubchanskii, E. A. Shapovalov, A. E. Zabolotin, K. Y. Guslienko, Th. Rasing, Nonlinear magneto-optical diffraction by twodimensional magnetic superstructures, Appl. Phys. B 74 (2002) 711-714.

[6] N. N. Dadoenkova, I. L. Lyubchanskii, M. I. Lyubchanskii, E. A. Shapovalov, A. E. Zabolotin, Th. Rasing, Influence of magnetic field on nonlinear magneto-optical diffraction on two-dimensional hexagonal magnetic bubble lattice, J. Opt. Soc. Am. B 22 (2005) 215-219.

[7] S. V. Lazarenko, A. Kirilyuk, Th. Rasing, J. C. Lodder, Linear and nonlinear magneto-optical diffraction from one-dimensional periodic structures, J. Appl. Phys. 93 (2003) 7903-7905.

[8] V. Berger, Nonlinear photonic crystals, Phys. Rev. Lett. 81 (1998) 4136-4139.

[9] A. M. Malvezzi, F. Cattaneo, G. Vecchi, M. Falasconi, G. Guizzetti, L. C. Andreani, F. Romanato, L. Businaro, E. Di Fabrizio, A. Passaseo, M. De Vittorio, Second-harmonic generation in reflection and diffraction by GaAs photonic-crystal waveguide, J. Opt. Soc. Am. B 19 (2002) 2122-2128.

[10] A. A. Fedyanin, O. A. Aktsipetrov, D. A. Kurdyukov, V. G. Golubev, M. Inoue, Nonlinear diffraction and second-harmonic generation enhancement in silicon-opal photonic crystals, Appl. Phys. Lett. 87 (2005) 151111(3).

[11] E. Estephan, D. Bajoni, M. B. Saab, T. Cloitre, R. Aulombard, C. Larroque, L.C. Andreani, M. Liscidini, A. M. Malvezzi, C. Gergely, Sensing by means of nonlinear pptics with functionalized GaAs/AlGaAs photonic crystals, Langmuir 26 (2010) 10373-10379.

[12] S. M. Saltiel, D. N. Neshev, R. Fischer, W. Krolikowski, A. Arie, Yu. S. Kivshar, Generation of second-harmonic conical waves via nonlinear Bragg diffraction, Phys. Rev. Lett. 100 (2008) 103902(4).

[13] S. M. Saltiel, D. N. Neshev, W. Krolikowski, A. Arie, O. Bang, Yu. S. Kivshar, Multiorder nonlinear diffraction in frequency doubling processes, Opt. Lett. 34 (2009) 848-850.

[14] J. W. Tucker, V. W. Rampton, Hypersound in Solid State Physics, North-Holland, Amsterdam, 1972. 
[15] D. F. Nelson and M. Lax, Theory of acoustically induced optical harmonic generation, Phys. Rev. B 3 (1971) 2795-2812.

[16] F. Barocchi, Multiphoton-phonon interactions and higher-order Brillouin scattering: (Steady-state gain amplification), Opt. Comm. 3 (1971) 189-193.

[17] B. F. Levine, Calculation of the direct acoustically induced optical harmonic generation coefficient, Phys. Rev. Lett. 30 (1973) 171-174.

[18] B. V. Bokut', N. A. Khilo, V. I. Kondratenko, P. A. Khilo, Optical second harmonic generation under collinear light diffraction on ultrasound, Proceedings of the Academy of Sciences of the Byelorussian SSR 27, (1983), 114-116 (in Russian).

[19] G. D. Boyd, F. R. Nash, D. F. Nelson, Observation of acoustically induced phase-matched optical harmonic generation in GaAs, Phys. Rev. Lett. 24 (1970) 1298-1301.

[20] J.-W. Jeong, S.-C. Shin, I. L. Lyubchanskii, V. N. Varyukhin, Strain-induced three-photon effects, Phys. Rev. B 62, 13455-13463 (2000).

[21] I. L. Lyubchanskii, N. N. Dadoenkova， M. I. Lyubchanskii， Th. Rasing， J.-W. Jeong, S.-C. Shin, Second-harmonic generation from realistic film-substrate interfaces: The effects of strain, Appl. Phys. Lett. 76 (2000) 1848-1850.

[22] J. Reif, R. Schmid, Th. Schneider, D. Wolfframm, Nonlinear optical characterization of the surface of silicon wafers: In-situ detection of external stress, Solid-State Electronics 44 (2000) 809-813.

[23] J.-W. Jeong, S.-C. Shin, N. N. Dadoenkova, I. L. Lyubchanskii, V. K. Valev, Th. Rasing, Direct observation of controlled strain-induced second harmonic generation in a $\mathrm{Co}_{0.25} \mathrm{Pd}_{0.75}$ thin film on a $\mathrm{Pb}(\mathrm{ZrTi}) \mathrm{O}_{3}$ substrate, Appl. Phys. Lett. 90 (2007) 044108(3).

[24] P. Kumar, A. I. Maydykovskiy, M. Levy, N. V. Dubrovina, O. A. Aktsipetrov, Second harmonic generation study of internally generated strain in bismuth-substituted iron garnet films, Opt. Express 18 (2010) 1076-1084.

[25] C. Schriever, C. Bohley, R. B. Wehrspohn, Strain dependence of second harmonic generation in silicon, Opt. Lett. 35 (2010) 273-275; C. Schriever, C. Bohley, R. B. Wehrspohn, Strain-induced nonlinear optics in silicon, in: M. Hanbücken, P. Müller, R.B. Werhspohn (Eds.), Mechanical Stress on the Nanoscale: Simulation, Material Systems and Characterization Techniques, Wiley, New York, 2011, pp. 333-355.

[26] M. Cazzanelli, F. Bianco, E. Borga, G. Pucker, M. Ghulinyan, E. Degoli, E. Luppi, V. Véniard, S. Ossicini, D. Modotto, S. Wabnitz, R. Pierobon, L. Pavesi, Second-harmonic generation in silicon waveguides strained by silicon nitride, Nature Materials 11 (2012) 148154.

[27] Y. R. Shen, The Principles of Nonlinear Optics, Wiley, New York, 1984. 
[28] D. L. Mills, Nonlinear Optics: Basic Concepts, Springer-Verlag, Berlin, 1991.

[29] C. Kittel, Introduction to Solid State Physics, eighth ed., Wiley, New York, 2004.

[30] J. A. Rogers, A. A. Maznev, M. J. Banet, and K. A. Nelson, Optical generation and characterization of acoustic waves in thin films: Fundamentals and applications, Annu. Rev. Mater. Sci. 30 (2000) 117-157. 
Figure captions for the manuscript "Nonlinear Optical Diffraction by Standing Acoustic Waves in a GaAs film" by N.A. Shevchenko et al.

Fig. 1. Schematic of nonlinear acousto-optical diffraction by standing acoustic waves in the transmission geometry.

Fig. 2. Dependence of the maximum number of observable diffraction orders upon incidence angle $\varphi_{\omega}$ for NOD due to standing L-acoustic waves (a) and T-acoustic waves (b) at various acoustic frequencies.

Fig. 3. Angular dependencies of the diffraction efficiency of the first order of NOD induced by a standing acoustic wave in GaAs for the following geometries: (a) and (b) represent NOD by a standing $L$-acoustic wave for the $s(\omega) \rightarrow p(2 \omega)$ and $p(\omega) \rightarrow p(2 \omega)$ diffractions, respectively; (c), (d), (e) and (f) correspond to NOD by a standing $T$-acoustic wave for the $s(\omega) \rightarrow s(2 \omega)$, $s(\omega) \rightarrow p(2 \omega), \quad p(\omega) \rightarrow s(2 \omega)$, and $p(\omega) \rightarrow p(2 \omega)$ diffractions, respectively.

In all cases, the standing wave frequency is $F_{L, T}=250 \mathrm{MHz}$, the intensity of the acoustic wave is equal to $30 \mathrm{~W} / \mathrm{cm}^{2}$, the diameter of the laser spot is $400 \mu \mathrm{m}$, and the thickness of the crystalline film is $0.5 \mathrm{~mm}$. 


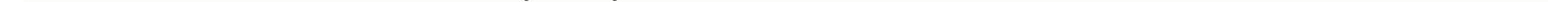




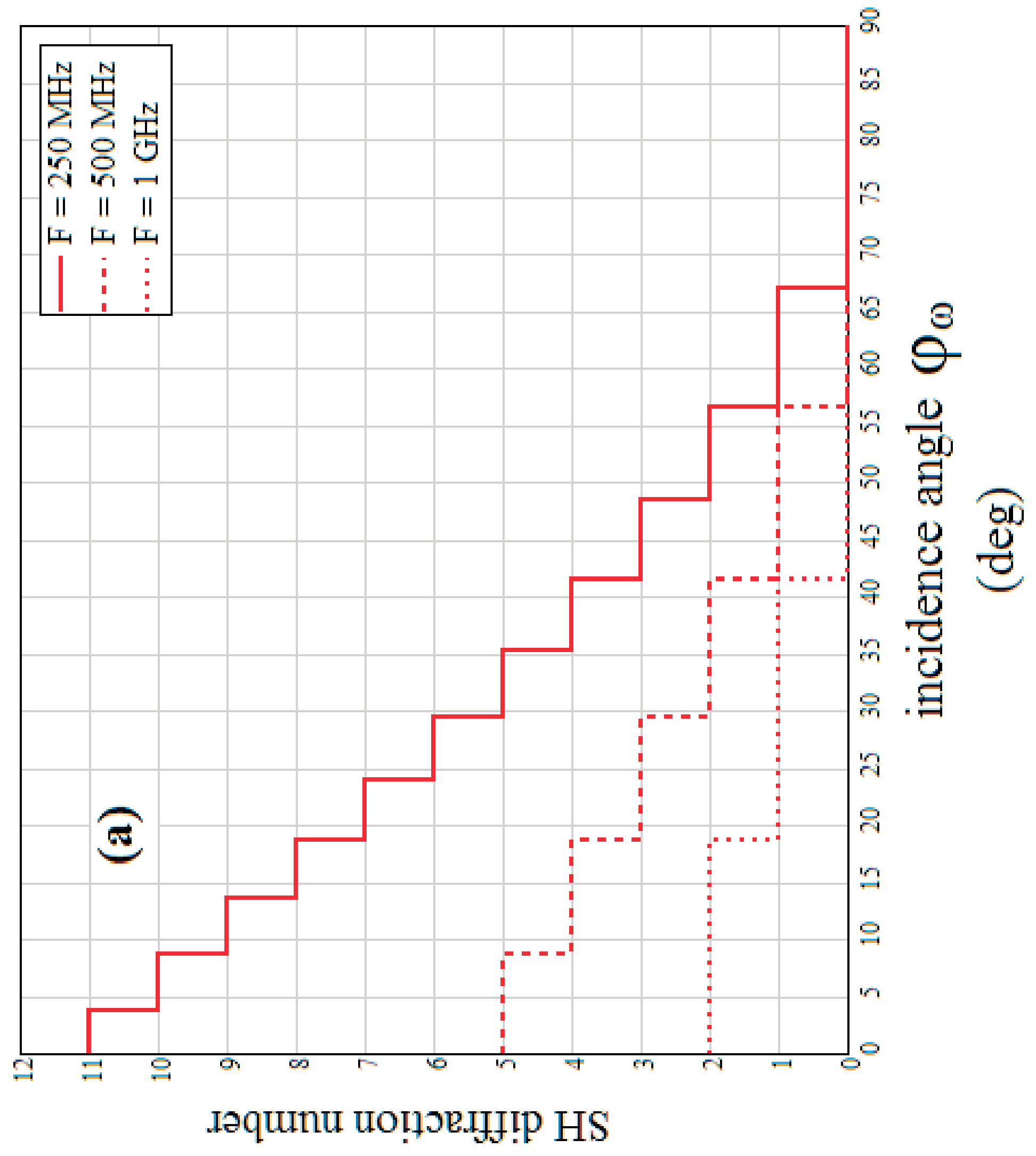




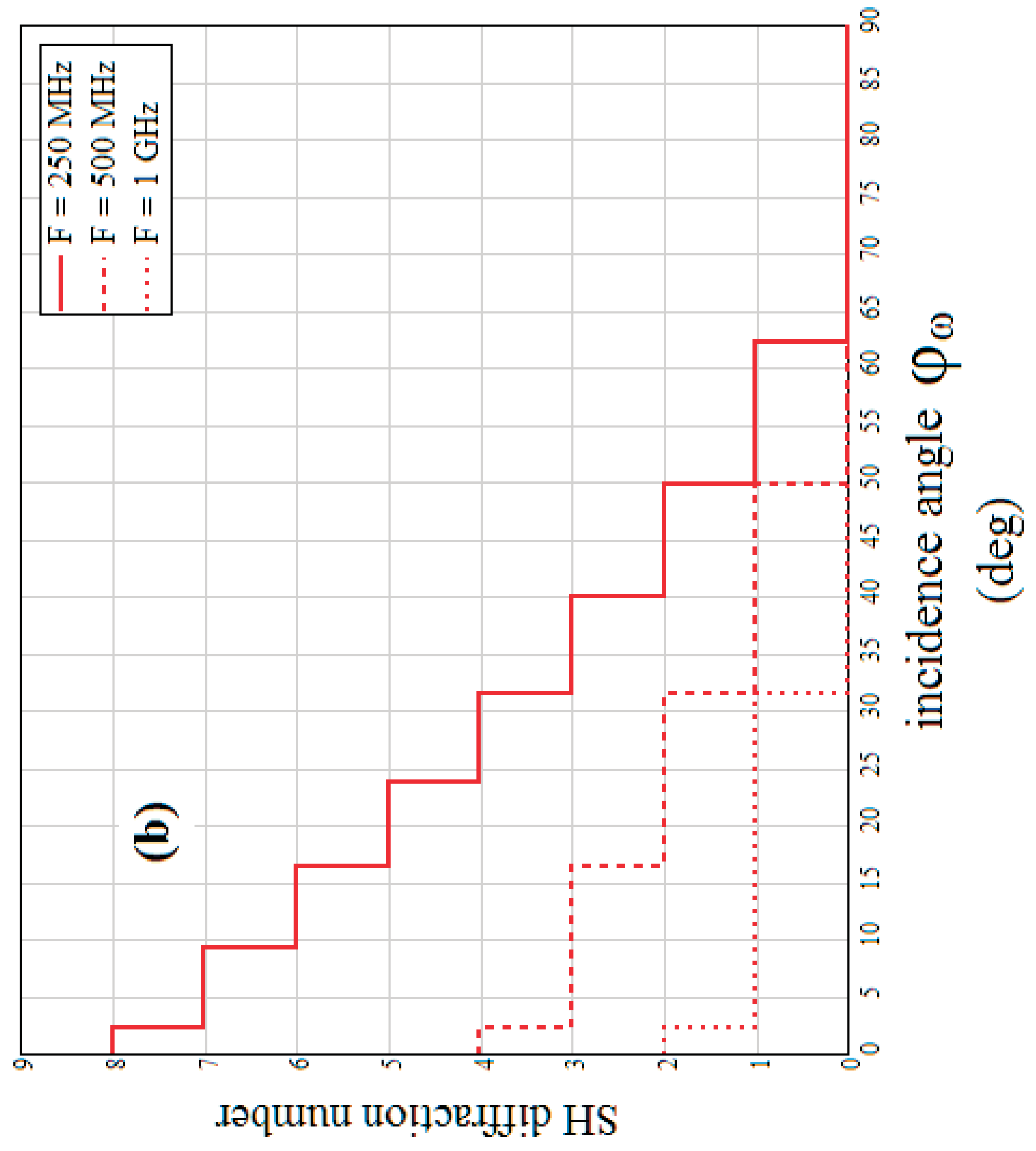




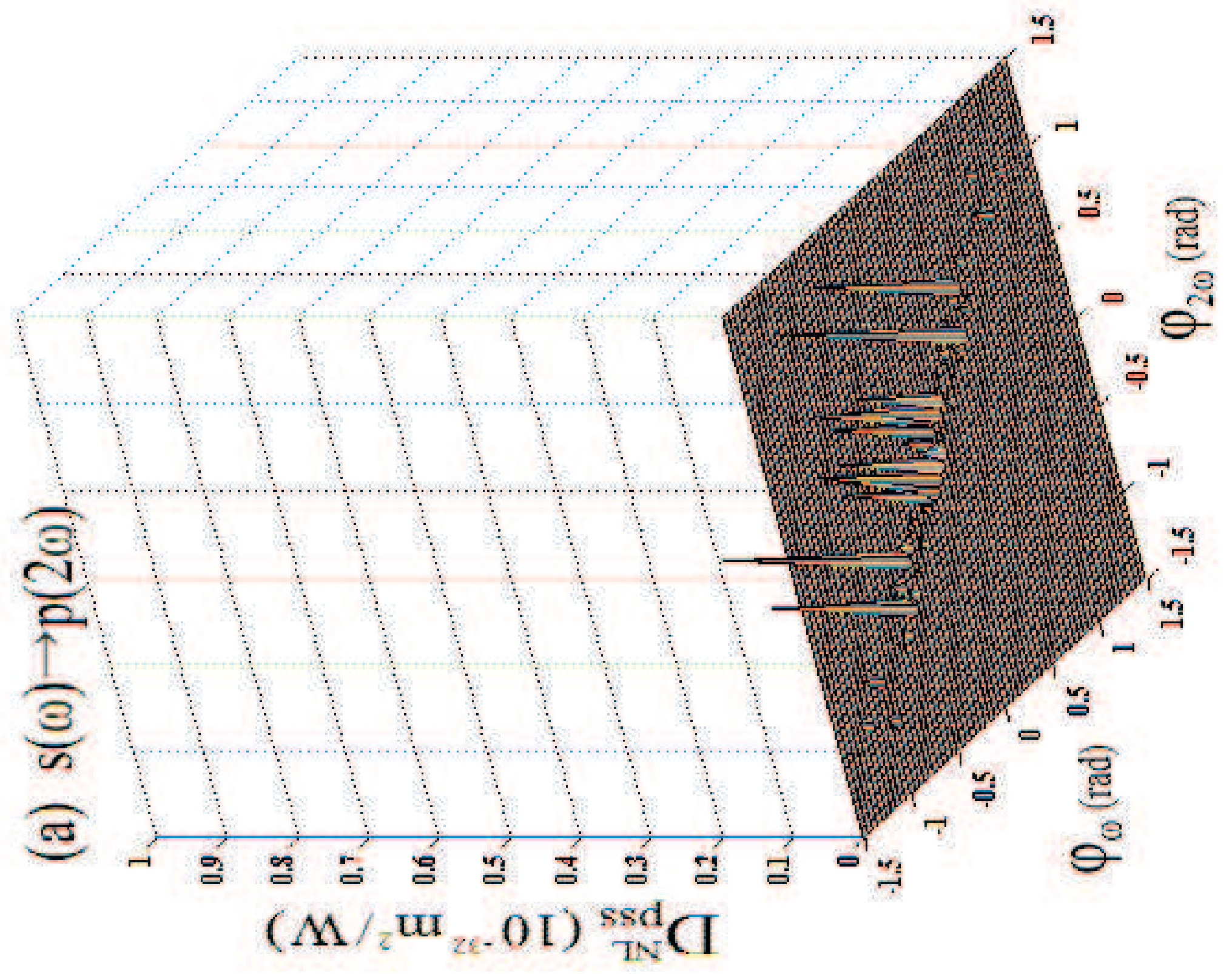




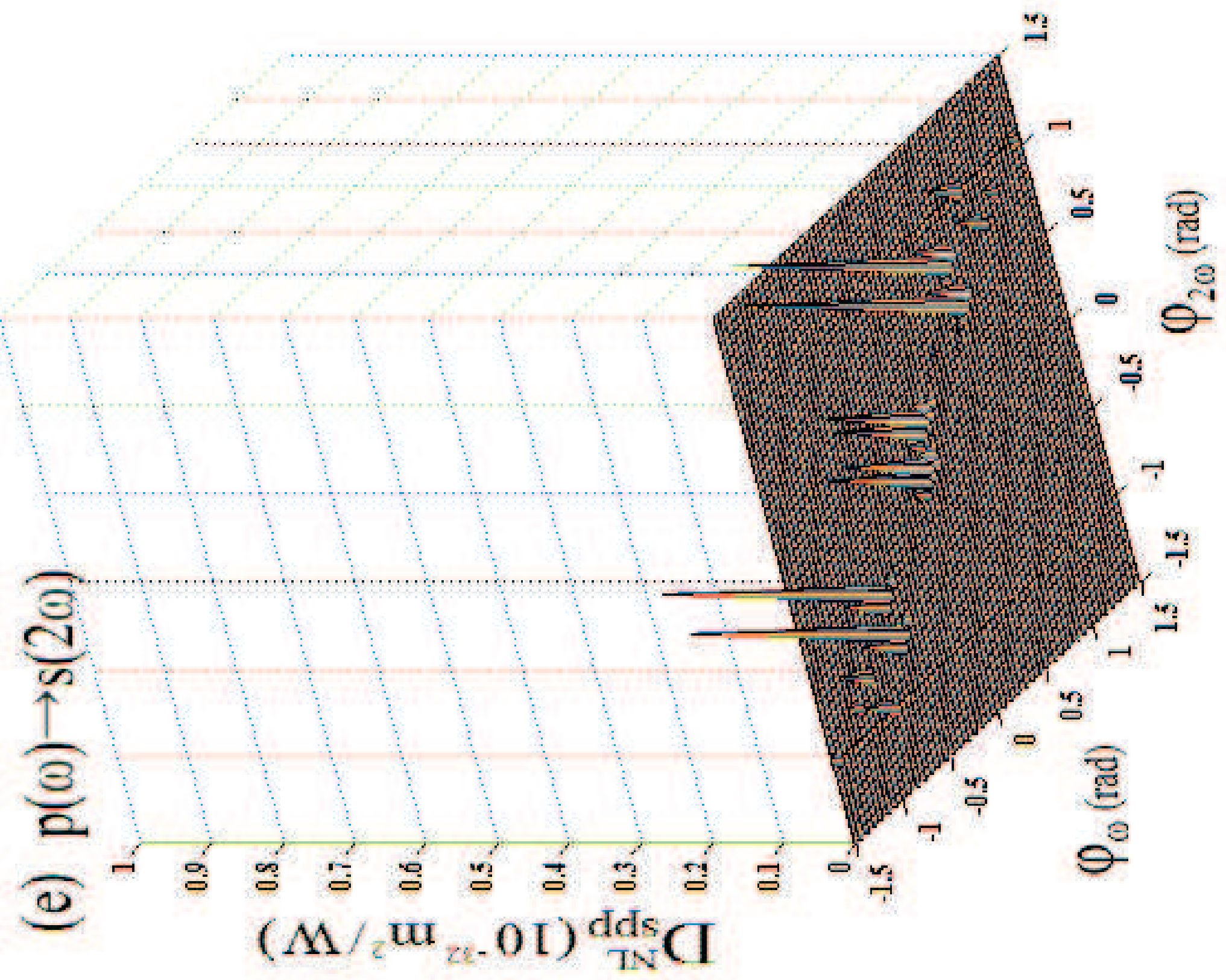




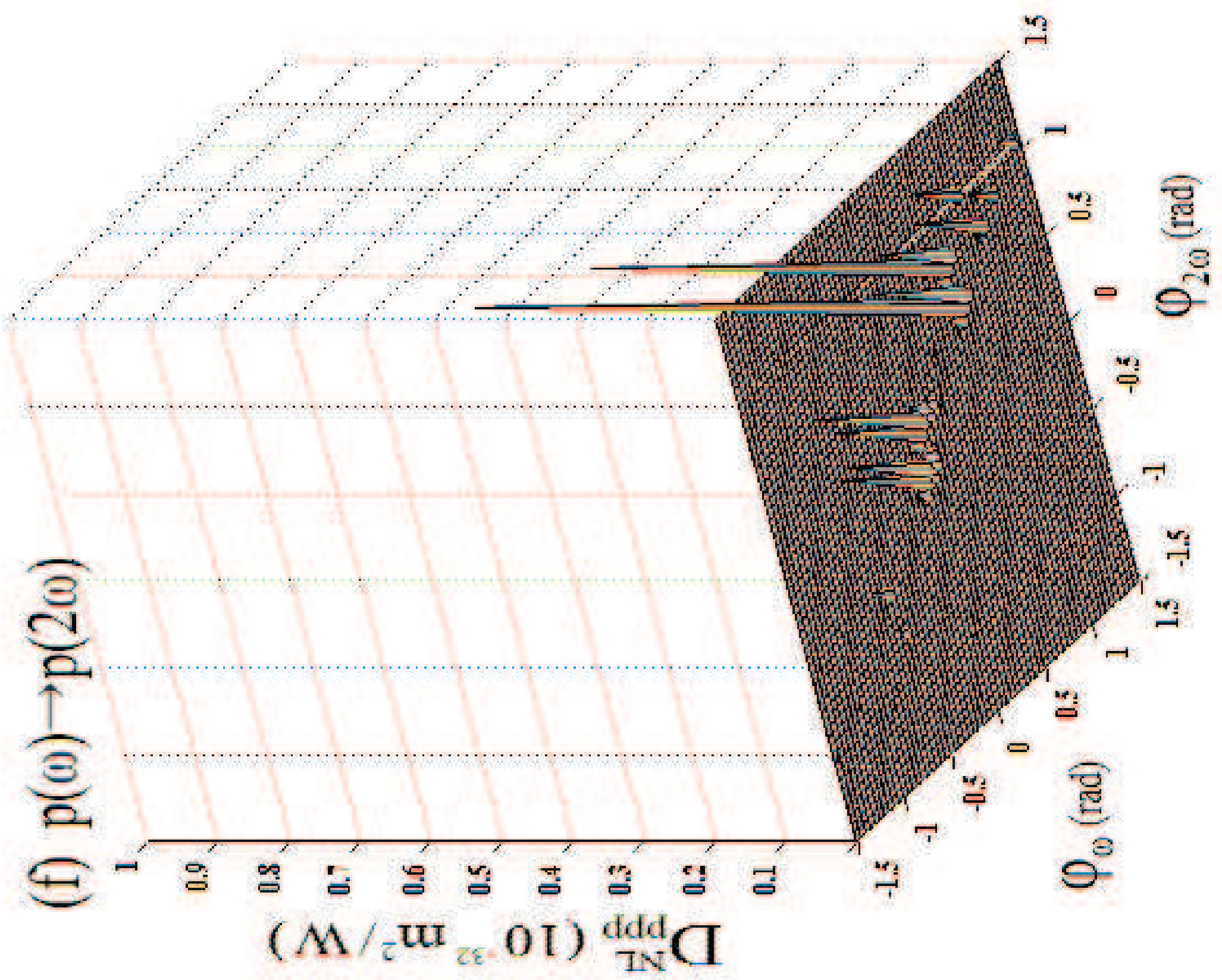

